\title{
Convex Polytopic Modeling of Diabetes Mellitus: A Tensor Product based approach
}

\author{
Levente Kovács and György Eigner \\ Research and Innovation Center of Óbuda University, \\ Physiological Controls Group, Óbuda University, Budapest, Hungary \\ Email: \{kovacs.levente,eigner.gyorgy\}@nik.uni-obuda.hu
}

\begin{abstract}
Tensor Product (TP) transformation based modeling and control can be useful in biomedical engineering, since complex nonlinear control tasks can be handled easier with it. Moreover, the modeling approach can handle the Linear Parameter Varying (LPV) models and produces a tensor based system description, which can be used during Linear Matrix Inequality (LMI) based controller design. The TP property makes the usability of the method beneficial as LMI connected techniques allows using the Lyapunov theorems. The aim of the current work is to demonstrate the usability of TP models in biomedical applications, i.e. diabetes modeling. The core model, the minimal model is investigated and simulation results are presented under Matlab.
\end{abstract}

Index terms-TP modeling, Tensor Product transformation, LPV modeling, modeling of diabetes mellitus, T1DM model, T2DM model

\section{INTRODUCTION}

Mathematical modeling and in-silico simulation has great importance in case of physiological modeling. If the models describe internal relationships, their investigation increases the complexity of the problem as they represent living organisms with continuously changing parameters [1]. In the recent years, several advanced modeling techniques appeared in the biomedical engineering topic regarding modern control engineering methodologies. Through these methods, not only the "pure" dynamics of the given processes can be taken into account, but also uncertainties coming from subject (patient) variability [2]. A good example of that is the modeling of diabetes mellitus (DM) [3].

From modeling and controller design point of view, the process of DM is highly unfavorable: a nonlinear process, which suffers from time-to-time inter- and intra-patient variabilities, periodical signals (feed and insulin intakes, internal insulin secretion) and time-delays; moreover, several internal processes cannot be directly observed in real life. DM models try to reflect these nonlinear properties taking into account the time-lags as well. However, inter-and intra-patient variabilities are hard to handle as they could occur in the parameters of the variables [3]. On the other way, from control design point of view, complex models would be beneficial, but their clinical applicability will be questionable [4].
Therefore, in the last two decades several possibilities have been investigated to handle the above described problems. From control modeling point of view Linear Paramter Varying (LPV) methodology proved to be a suitable choice transforming the original nonlinear model into a linear one approximation (linearization) [5], [6]. Recently, TP-based modeling [7][9] gives another tool of transforming the nonlinear model in a linear form without approximation. The aim of TPmodeling is to realize TP-model objects. These are beneficial because the linear controller design theorems can be adapted to them during the controller design. The resulting model and controller is the convex combination of the TP-model and controller objects. Different uncertainties can be included to the TP-models, moreover, these models are optimized for LMI based controller design as well [9], [10].

In this paper, we analyze a special form of a reference DM model used for both Type 1 and Type 2 DM (T1DM and T2DM) [11], by applying the TP methodology. The paper is structured, as follows: first, we introduce the TP modeling approach, then we present the DM model and the control optimized derivation of it. Section V investigates robustness improvement of the given model, followed by the validation of the model. Finally, conclusions are presented together with future work possibilities.

\section{THE TP MODEL TRANSFORMATION}

With the TP model transformation it is possible to transform given functions into TP model functions [7], [12]. The occurring TP model function is based on a multidimensional tensor product, where a high-order core tensor structure is multiplied by different weighting functions with appropriate dimensions. Since, the quasi-LPV (qLPV) models can be described with qLPV functions, the TP model form of given qLPV models can be calculated [9], [13]. The realized TP model approximates the original model with eligible accuracy. In this way the TP transformation can be combined with LMI-based control design techniques as it provides appropriate way for convex hull manipulation of polytopic structures.

A possible general parameter-dependent qLPV model (with $k$ states, $m$ inputs and $l$ outputs) can be described as follows:

$$
\begin{aligned}
\dot{\mathbf{x}}(t) & =\mathbf{A}(\mathbf{p}(t)) \mathbf{x}(t)+\mathbf{B}(\mathbf{p}(t)) \mathbf{u}(t) \\
\mathbf{y}(t) & =\mathbf{C}(\mathbf{p}(t)) \mathbf{x}(t)+\mathbf{D}(\mathbf{p}(t)) \mathbf{u}(t)
\end{aligned}
$$




$$
\mathbf{S}(\mathbf{p}(t))=\left(\begin{array}{ll}
\mathbf{A}(\mathbf{p}(t)) & \mathbf{B}(\mathbf{p}(t)) \\
\mathbf{C}(\mathbf{p}(t)) & \mathbf{D}(\mathbf{p}(t))
\end{array}\right)
$$

where $\mathbf{A}(\mathbf{p}(t)) \in \mathbb{R}^{k \times k}$ represents the state matrix, $\mathbf{B}(\mathbf{p}(t)) \in$ $\mathbb{R}^{k \times m}$ the input matrix, $\mathbf{C}(\mathbf{p}(t)) \in \mathbb{R}^{l \times k}$ the output matrix and $\mathbf{D}(\mathbf{p}(t)) \in \mathbb{R}^{l \times m}$ the forward matrix. $\mathbf{u}(t) \in \mathbb{R}^{m}$ represents the input vector, $\mathbf{y}(t) \in \mathbb{R}^{l}$ the output vector and $\mathbf{x}(t) \in \mathbb{R}^{k}$ states for the state vector. $\mathbf{S}(\mathbf{p}(t)) \in \mathbb{R}^{(k+l) \times(k+m)}$ is the parameter dependent system matrix, which equivocally determines the qLPV system itself.

$\mathbf{p}(t) \in \Omega \in \mathbb{R}^{N}$ represents the time dependent parameter vector, where $\Omega=\left[p_{1, \text { min }}, p_{1, \max }\right] \times\left[p_{2, \text { min }}, p_{2, \max }\right] \times \ldots \times$ $\left[p_{N, \min }, p_{N, \max }\right] \in \mathbb{R}^{N}$ realizes a closed hypercube in an $N$ dimensional hyperspace determined by the extremes of the elements of the parameter vector [9], [14]. Hence, the finite element polytopic model describes the qLPV model inside the closed hypercube as follows:

$$
\mathbf{S}(\mathbf{p}(t))=\sum_{r=1}^{R} w_{r}(p(t)) \mathbf{S}_{r}
$$

In this configuration, the $\mathbf{S}(\mathbf{p}(t))$ can be calculated as a convex combination of the LTI vertex system for each $\mathbf{p}(t) \in \Omega$. From here, the TP based polytopic finite element model is determined through a "sampling" on the parameter space [9], [15]:

$$
\mathbf{S}(\mathbf{p}(t))=\sum_{i_{1}=1}^{I_{1}} \sum_{i_{2}=1}^{I_{2}} \ldots \sum_{i_{N}=1}^{I_{N}} \prod_{n=1}^{N} w_{n, i_{n}}\left(p_{n}(t)\right) \mathbf{S}_{i_{1}, i_{2}, \ldots, i_{N}}
$$

which can be described in compact form, as follows:

$$
\mathbf{S}(\mathbf{p}(t))=S \underset{\mathrm{n}=1}{\mathrm{~N}} \mathbf{w}_{n}\left(p_{n}(t)\right),
$$

where the $S \in \mathbb{R}^{I_{1} \times I_{2} \times \ldots \times I_{N} \times(k+l) \times(k+m)}$ coefficient tensor is created from the LTI vertex systems $\mathbf{S}_{i_{1}, i_{2}, \ldots, i_{N}}$ and $\mathbf{w}_{n}\left(p_{n}(t)\right)$ vector consist of $w_{n, i_{n}}\left(p_{n}(t)\right)\left(i_{n}=1 \ldots I_{N}\right)$ continuous weighting functions. Hence, the convexity criteria for a given TP model is satisfied if the following statements are true for the weighting functions:

$$
\begin{aligned}
& \forall n, i, p_{n}(t): w_{n, i_{n}}\left(p_{n}(t)\right) \in[0,1] \\
& \forall n, p_{n}(t): \sum_{i=1}^{I_{n}} w_{n, i_{n}}\left(p_{n}(t)\right)=1
\end{aligned} .
$$

In this study, we use the Minimal Volume Simplex (MVS) type convex hull for the TP type polytopic qLPV model [9], [10]:

$$
\mathbf{S}(\mathbf{p})=S \underset{\mathrm{n}=1}{\mathrm{~N}} \mathbf{w}^{(n)}\left(p_{n}\right)
$$

Here, the core tensor $S \in \mathbb{S}^{J_{1} \times \ldots \times J_{N}}$ is realized from the $\mathbf{S}_{j_{1}, \ldots, j_{N}}$ matrices. In this way, the $(S)_{j_{n}=j} n$-mode subtensors evolve a minimal volume bounding simplex for the $S \times{ }_{n} \mathbf{w}_{j n}^{(n)}\left(p_{n}\right)$ trajectory over $n=1 . . N$. More detailed explanation can be found in [9], [10], [16], [17].
In the current study, we utilized the TP Toolbox ${ }^{\circledR}$, which is a MATLAB extension and provides convenient solution for using TP-based approaches. The TP toolbox is available at [18].

\section{INVESTIGATED Minimal MODEL}

We used a modified version of the Minimal Model in this study, which is appropriate to describe the T1DM and T2DM cases, respectively [11]. The model equations are the following:

$$
\begin{array}{r}
\dot{G}(t)=-\left(p_{1}+X(t)\right) G(t)+p_{1} G_{B}+d(t) \\
\left.\dot{X}(t)=-p_{2} X(t)\right)+p_{3}\left(I(t)-I_{B}\right) \\
\dot{I}_{T 2 D M}(t)=\left\{\begin{array}{r}
\gamma(G(t)-h) t-n\left(I(t)-I_{B}\right)+u(t) \\
\text { for } G(t)-h>0 \\
-n\left(I(t)-I_{B}\right)+u(t) \\
\text { for } G(t)-h \leq 0
\end{array}\right. \\
\dot{I}_{T 1 D M}(t)=-n\left(I(t)-I_{B}\right)+u(t)
\end{array}
$$

The model has three states: $G(t)[\mathrm{mg} / \mathrm{dL}]$ the blood glucose concentration, which represents at the same time the output of the model; $X(t)[1 / \mathrm{min}]$ the insulin-excitable tissue glucose uptake activity, and $I(t)[\mu \mathrm{U} / \mathrm{mL}]$ the blood insulin concentration. The model has two inputs: the external insulin intake $u(t)[\mu \mathrm{U} / \mathrm{mL} / \mathrm{min}]$ and the glucose intake $d(t)[\mathrm{mg} / \mathrm{dL} / \mathrm{min}]$.

The T2DM state is described by (7c), where the internal insulin production is only possible when the $G(t)$ is higher than a threshold $h$. The simplified T1DM case is represented by $(7 \mathrm{~d})$, where is no internal insulin production.

In this study we used the following parameter set: $G_{b}=110$ $\mathrm{mg} / \mathrm{dL}, I_{b}=1.5 \mu \mathrm{U} / \mathrm{mL}, p_{1}=0.0281 / \mathrm{min}, p_{2}=0.025$ $1 / \mathrm{min}, p_{3}=0.00013 \mathrm{~min}^{-2} /(\mu \mathrm{U} / \mathrm{mL}), n=0.231 / \mathrm{min}$, $h=130 \mathrm{mg} / \mathrm{dL}, \gamma=0.01(\mu \mathrm{U} / \mathrm{mL}) /(\mathrm{mg} / \mathrm{dL}) / \mathrm{min}$. These parameters belong to a real patient based on [11]. As the goal is to demonstrate the applicability of TP-model approach we did not distinguish the different cases on the parameter level; hence, to prove that the method works regardless from the used parameter sets.

\section{Possible DeVIATION-BASED QLPV AND TP MODElS}

A popular modeling approach is the control oriented deviation based modeling [2], [16]. In this case the models describe the dynamics of the process and the deviation from a possible equilibrium; further, the control goal is to avoid or eliminate the deviation from this equilibrium. The TP-based techniques using Linear Matrix Inequalities (LMI) can provide good performance beside such kind of models [10].

First, we investigated the steady-state conditions in a possible equilibrium. We selected $G_{d}=90$ and $u_{d}=0$ as steadystate values (the blood glucose concentration is $90 \mathrm{mg} / \mathrm{dL}$ and there is no external insulin intake). Moreover, we considered that $G_{d} \neq G_{B}$. From here, the other necessary steady-state 
values can be calculated by rearranging (7a)-(7d). It should be noted that $h>G_{d}$, so (7c) and (7d) has the same $I_{d}$ :

$$
\begin{gathered}
I_{d}=\frac{n I_{b}+u_{d}}{n} . \\
X_{d}=\frac{p_{3}}{p_{2}} I_{d} . \\
d_{d}=\left(p_{1}+X_{d}\right) G_{d}-p_{1} G_{B} .
\end{gathered}
$$

With the calculated steady-state values, the deviation based model can be derived from the model equations, as follows. First, the $\Delta G(t)$ has been determined. Note that since the $G(t)$ is measurable in real life, we tried to realize a form where only $G(t)$ appears in the state matrix of the deviation based model:

$$
\begin{aligned}
& \Delta \dot{G}(t)=\dot{G}(t)-0= \\
& -\left(p_{1}+X(t)\right) G(t)+p_{1} G_{B}+d(t)- \\
& {\left[-\left(p_{1}+X_{d}\right) G_{d}+p_{1} G_{B}+d_{d}\right]=} \\
& -p_{1}\left(G(t)-G_{d}\right)+\left(d(t)-d_{d}\right)-X(t) G(t)+X_{d} G_{d}=\text {. } \\
& -p_{1} \Delta G(t)+\Delta d(t)- \\
& X(t) G(t)+X_{d} G_{d}+X_{d} G(t)-X_{d} G(t) \\
& \Delta \dot{G}(t)=-\left(p_{1}+X_{d}\right) \Delta G(t)+\Delta d(t)-G(t) \Delta X(t)
\end{aligned}
$$

Due to lack of space, we did not detail the derivations of further deviation based forms or state variables. However, we used the same tools as in case of (11) resulting for $\Delta X$ as follows:

$$
\Delta \dot{X}(t)=-p_{2} \Delta X(t)+p_{3} \Delta I(t) .
$$

In case of $\Delta I$ we had to differentiate the deviation based forms for T1DM $\left(I_{T 1 D M}\right)$ and T2DM $\left(I_{T 2 D M}\right)$ :

$$
\Delta \dot{I}_{T 1 D M}(t)=-n \Delta I(t)+\Delta u(t)
$$

and

$$
\Delta \dot{I}_{T 2 D M}(t)=\left\{\begin{array}{c}
\gamma \frac{(G(t)-h)}{\Delta G(t)} \Delta G(t)-n \Delta I(t)+\Delta u(t) \\
-n \Delta I(t)+\Delta u(t) \quad \text { for } G(t)>h \\
\text { for } G(t) \leq h
\end{array}\right.
$$

A convenient solution results if we use the derived deviation based model in state-space form. In this case, the states should be $\Delta x(t)=[\Delta G(t), \Delta X(t), \Delta I(t)]^{T}$. Thus, the investigated qLPV models become as described in (15)(16). Applying the TP model transformation on these (having only one parameter), the general TP model structure becomes $\mathbf{S}(G(t))=S \times \mathbf{w}(G(t))$.

As a result, the variation of the obtained MVS type weighting functions can be presented on Fig. 1. In case of T1DM, the weighting function is linear, however, in T2DM case the weighting function is nonlinear because of the fraction in (14).
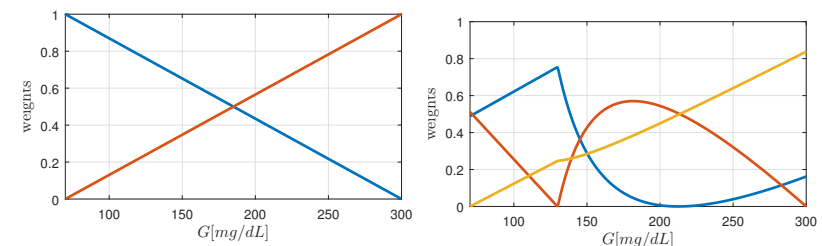

Figure 1. Weighting functions of the TP polytopic model; simple model case

It should be noted, that, $\Delta G(t)$ cannot be zero until the $G_{d}$ is lower than $h$.

\section{ROBUSTNESS OF THE MODELS}

In order to increase the robustness of the model (and the realizable controller based on the TP models) the most determinant model parameters should be investigated from the model output point of view. The TP transformation based modeling and control property is that the modeling and controller design can be coupled directly to LMI-based controller design methods. This coupling provides a unique way to increase the robustness through the elements of the parameter vector increasing the control performance. If, the parameter vector contains several parameters and the borders of them are given than the controller will be prepared for the varying of these parameters between the given borders.

The output of the model is the blood glucose level $G(t)$, the only measurable variable in real life circumstances. Thus, it is reasonable to investigate how model parameter variation affects $G(t)$. We applied simple perturbation analysis based investigation in order to identify the most determining model parameter. We used the non-normalized Root Mean Square Error (RMSE) to evaluate the results.

$$
\begin{aligned}
\Delta \dot{x}_{T 1 D M}(t) & =\left[\begin{array}{ccc}
-\left(p_{1}+X_{d}\right) & -G(t) & 0 \\
0 & -p_{2} & p_{3} \\
0 & 0 & -n
\end{array}\right] \Delta x(t)+\left[\begin{array}{l}
0 \\
0 \\
1
\end{array}\right] \Delta u(t)+\left[\begin{array}{l}
1 \\
0 \\
0
\end{array}\right] \Delta d(t) . \\
\Delta \dot{x}_{T 2 D M}(t) & =\left[\begin{array}{ccc}
-\left(p_{1}+X_{d}\right) & -G(t) & 0 \\
0 & -p_{2} & p_{3} \\
\gamma \frac{(G(t)-h)}{\Delta G(t)} t & 0 & -n
\end{array}\right] \Delta x(t)+\left[\begin{array}{l}
0 \\
0 \\
1
\end{array}\right] \Delta u(t)+\left[\begin{array}{l}
1 \\
0 \\
0
\end{array}\right] \Delta d(t) .
\end{aligned}
$$


The same investigation process was used both for T1DM and T2DM cases:

- Compare the output of the nominal model $G_{\text {orig }}\left(t_{i}\right)$ to the output of the perturbed model $G_{\text {pert }}\left(t_{i}\right), R M S E_{\text {param }}=$ $\sum_{t_{i}=0}^{T} \sqrt{G_{\text {orig }}\left(t_{i}\right)-G_{\text {pert }}\left(t_{i}\right)}$.

- Use a $\pm 35 \%$ perturbation in case of each parameter.

- Apply impulse input signals both for $\mathrm{CHO}$ and insulin inputs (Parameters: CHO: $d(t)=10 \mathrm{mg} / \mathrm{dL}$ over 6 minutes; insulin: $u(t)=20 \mathrm{uU} / \mathrm{mL}$ over 6 minutes ; injection time: beginning of simulation (minute 0 )). The simulation length was selected $T=100 \mathrm{~min}$.

Table I summarizes the results:

Table I

RESULTS OF THE RMSE-BASED INVESTIGATIONS.

\begin{tabular}{|c|c|c|c|}
\cline { 3 - 4 } \multicolumn{2}{c|}{} & \multicolumn{2}{c|}{ Type } \\
\hline Parameter & Perturbation & RMSE $_{T 1 D M}$ & RMSE $_{T 2 D M}$ \\
\hline \multirow{2}{*}{$p_{1}$} & $-35 \%$ & 8.2385 & 9.2256 \\
\cline { 2 - 4 } & $+35 \%$ & 5.5199 & 6.0665 \\
\hline \multirow{2}{*}{$p_{2}$} & $-35 \%$ & 11.0671 & 11.3595 \\
\cline { 2 - 4 } & $+35 \%$ & 7.5582 & 7.842 \\
\hline \multirow{2}{*}{$p_{3}$} & $-35 \%$ & 7.6965 & 7.5272 \\
\cline { 2 - 4 } & $+35 \%$ & 6.0048 & 5.8129 \\
\hline \multirow{2}{*}{$n$} & $-35 \%$ & 9.832 & 9.8148 \\
\cline { 2 - 4 } & $+35 \%$ & 5.832 & 5.834 \\
\hline \multirow{2}{*}{$h$} & $-35 \%$ & - & 3.0358 \\
\cline { 2 - 4 } & $+35 \%$ & - & 1.4574 \\
\hline \multirow{2}{*}{$\gamma$} & $-35 \%$ & - & 0.5817 \\
\cline { 2 - 4 } & $+35 \%$ & - & 0.5605 \\
\hline
\end{tabular}
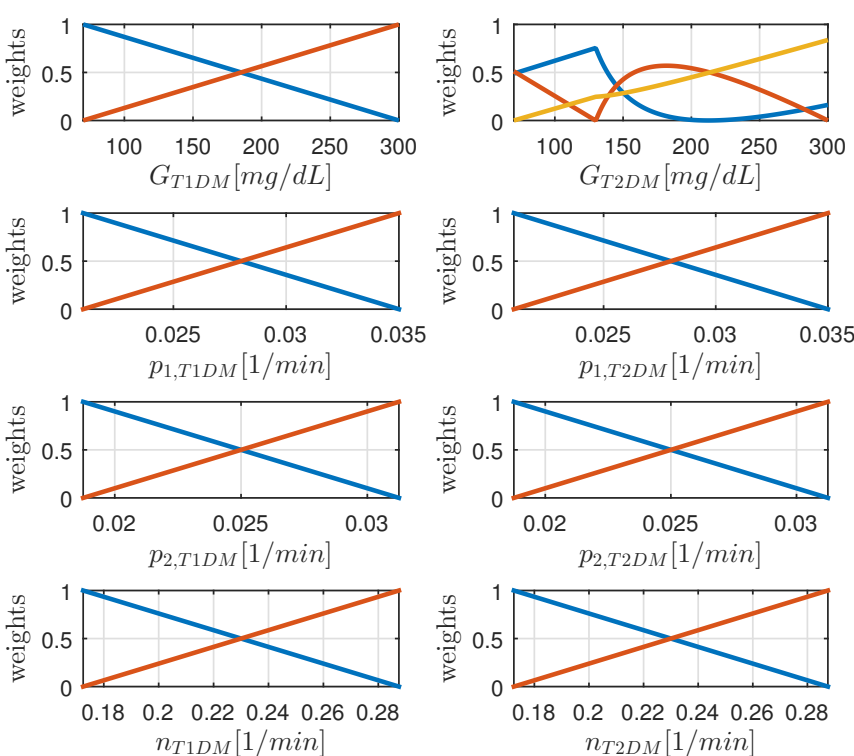

Figure 2. Weighting functions of the TP polytopic model; robust model case

Through this investigation it turned out that the most determining parameters to $G(t)$ are the $p_{1}, p_{2}$ and $n$. As the model is quite simple, each parameter variation may induce high perturbations that should be handled separately (our goal was finding a method appropriately providing the most important parameters). Hence, we have selected $p_{1}, p_{2}$ and $n$ as time varying parameters (beside $G(t)$ ) resulting a $4 D$ parameter space determined by the parameter vector $p_{4}(t)=\left[G(t), p_{1}(t), p_{2}(t), n(t)\right]^{T}$. The new elements are slowly changing in time, which allows handling them as constants. Naturally, the accurate values of them have to be updated after the identifications (done automatically).

The biggest advantage of this scenario relies in increasing the robustness of the controller in a special way. The $S$ core tensor provided by the TP model transformation can be used directly in LMI-based controller design. If the model parameters are handled as scheduling parameters, the controller will be prepared for the changing of these. In other words, as the core tensor is used during controller design and the core tensor contains the parameter dependencies, the controller could be even a simple state feedback one being handled inside the complex polytope.

Naturally, the TP model form is different in this case (having four scheduling parameters):

$$
\begin{aligned}
& \mathbf{S}\left(G(t), p_{1}, p_{2}, n\right)=S \stackrel{4}{\underset{\mathrm{n}=1}{\otimes}} \mathbf{w}_{n}\left(p_{n}(t)\right)= \\
& S \times_{1} \mathbf{w}_{1}(G(t)) \times_{2} \mathbf{w}_{2}\left(p_{1}\right) \times_{3} \mathbf{w}_{3}\left(p_{2}\right) \times_{4} \mathbf{w}_{4}(n)
\end{aligned}
$$

The MVS type weighting functions of the robustified TP models can be seen on Fig. 2.

\section{VALIDATION}

During the validation, we investigated the discrepancy between the original nonlinear models and their TP versions via the changing of their state variable over time during simulations. For evaluation we have used again the RMSEbased method.

We have applied symmetric impulse functions during the simulations both for the $\mathrm{CHO}$ and insulin inputs using the following protocol:

- $\mathrm{CHO}(d) 4 g$ over 5 min at every 50 min with $V_{g}=$ 11.2dL distribution volume, $A g=0.8$ utilization and molar weight $M w=180.12 \mathrm{~g} / \mathrm{mol}(\mathrm{CHO}=d * A g *$ $1000 / M w / V_{g}$; here: $28.2326 \mathrm{mg} / \mathrm{dL}$ over $5 \mathrm{~min}$ at every 50 min);

- insulin (u) $0.5 U$ over $2 \mathrm{~min}$ at every $50 \mathrm{~min}$ with $V_{i}=$ $8.4 d L$ distribution volume (insulin $=u * 1000 / V_{i}$; here $59.5238 \mu U / m L$ over $2 \mathrm{~min}$ at every $50 \mathrm{~min}$ ).

Corresponding to the reality, the input functions have impulse nature. However, they are unfavorable because of the higher amplitude and shorter time period occurred through real input signals. This is the reason why the above mentioned protocol was used ensuring that the TP model works under all circumstances.

Table II shows the results of the RMSE-based comparison between the state variables of the original nonlinear model and the realized TP models in simple model case, where only the $G(t)$ was the scheduling parameter. The first row describes 
the comparison between the original T1DM model and the TP version of it, while the second row presents the comparison between the original T2DM model and the TP version of the given model. We used high sampling density in the parameter domain. The borders of the domain were $70-300 \mathrm{mg} / \mathrm{dL}$ (as it can see on the horizontal axis of Fig. 1.

In both cases, beside the given inputs and initial values the TP models "mimic" the original nonlinear models with high precision (only numerical errors occured, i.e. magnitude lower than $10^{-8}$ ). Fig. 3 illustrates the obtained results. Negligible difference can be observed between the original model and the TP ones.

Table III represents the results of the RMSE-based comparison between the state variables of the original nonlinear model and the realized TP models in robust model case (the parameter vector contains four scheduling variables $p(t)=$ $\left.\left[G(t), p_{1}, p_{2}, n\right]^{T}\right)$. We applied again a high sampling density in the parameter domain (301 for $G$ and 11 for $p_{1}, p_{2}$ and $n$ ). The borders of the domains were set again $70-300 \mathrm{mg} / \mathrm{dL}$ for $G$, and $\pm 25 \%$ of the nominal $p_{1}, p_{2}$ and $n$ values (Fig. 2). Similar to the previously presented case the same inputs have been used for initial values.

With simple randomization, we investigated several parameter configurations for $p_{1}, p_{2}$ and $n$ inside the parameter ranges. Three specific cases (where we have found the highest errors) are highlighted founding. The given $p_{1}, p_{2}$ and $n$ parameters and the belonging data can be found in Table III. The comparisons have similar meanings as previously: the first row describes the RMSE between the original nonlinear T1DM model and the TP models, while the second row represents the RMSE between the original nonlinear T2DM model and the TP models. The highest errors in each case occur in the $G$ state as a natural consequence of the nonlinear attitude of the given weighting function (see the second column in the first row on Fig. 2). However, we found that the error can be tolerated being lower than 1 over the $300 \mathrm{~min}$ long simulation. Table III results are connected to the simulations of Fig. 4. One can see that the small deviation occured did not cause significant error in the dynamics of the models. The upper row describes the state variable of the T1DM models (original nonlinear and TP version) with the occurring error in time. The lower row presents the same comparison, however, for the T2DM models. It can be seen that the error has a "saturation" and the dynamics follow the dynamics of the state variables.

\section{REFERENCES}

[1] J. Bronzino and D. Peterson, The Biomedical Engineering Handbook, 4th ed. Boca Raton, Florida, USA: CRC Press, 2015.

[2] W. Levine, Ed., The Control Engineering Handbook, 2nd ed. Boca Raton, USA: CRC Press, Taylor and Francis Group, 2011.

[3] V.N. Shah, A. Shoskes, B. Tawfik, and S.K. Garg, "Closed-loop system in the management of diabetes: Past, present, and future," Diabetes Technol The, vol. 16, no. 8, pp. 477-490, 2014.

[4] J.T. Sorensen, "A physiologic model of glucose metabolism is man and
Table II

RESULTS OF THE RMSE-BASED INVESTIGATIONS; SIMPLE MODEL CASE. INITIAL VALUES: $G_{0}=100, X_{0}=0, I_{0}=11.5$; SIMULATION LENGTH: 150min; SAMPLING DENSITY IN THE PARAMETER DOMAIN: 301.

\begin{tabular}{|c|c|c|c|}
\cline { 2 - 4 } \multicolumn{1}{c|}{} & \multicolumn{3}{c|}{ Original model } \\
\hline $\mathrm{TP}_{\mathrm{T} 1 \mathrm{DM}}$ & $2.984 \mathrm{e}-13$ & $\mathbf{X}$ & $\mathbf{I}$ \\
\hline $\mathrm{TP}_{\mathrm{T} 2 \mathrm{DM}}$ & $1.477 \mathrm{e}-8$ & $8.372 \mathrm{e}-17$ & $2.22 \mathrm{e}-16$ \\
\hline
\end{tabular}

Table III

RESUlTS OF THE RMSE-BASED INVESTIGATIONS; ROBUST MODEL CASE. INITIAL VALUES: $G_{0}=100, X_{0}=0, I_{0}=11.5$; SIMULATION LENGTH: 300min; SAMPLING DENSITY IN THE PARAMETER DOMAIN: $G-301$, $p_{1}-11, p_{2}-11$ AND $p_{3}-11$.

\begin{tabular}{|c|c|c|c|}
\hline & \multicolumn{3}{|c|}{ Original model } \\
\hline & \multicolumn{3}{|c|}{$p_{1}=0.0266, p_{2}=0.0258, n=0.2231$} \\
\hline & $\mathbf{G}$ & $\mathbf{X}$ & $T$ \\
\hline $\mathrm{TP}_{\mathrm{T} 1 \mathrm{DM}}$ & 0.877 & $5.898 \mathrm{e}-16$ & 0 \\
\hline \multirow[t]{3}{*}{$\mathrm{TP}_{\mathrm{T} 2 \mathrm{DM}}$} & 0.877 & $1.9646 \mathrm{e}-16$ & $2.442 \mathrm{e}-15$ \\
\hline & \multicolumn{3}{|c|}{$p_{1}=0.0280, p_{2}=0.025, n=0.23$} \\
\hline & G & $\mathbf{X}$ & $T$ \\
\hline $\mathrm{TP}_{\mathrm{T} 1 \mathrm{DM}}$ & $1.165 \mathrm{e}-12$ & $5.8417 \mathrm{e}-16$ & 0 \\
\hline \multirow[t]{3}{*}{$\mathrm{TP}_{\mathrm{T} 2 \mathrm{DM}}$} & $7.1 \mathrm{e}-13$ & $2.688 \mathrm{e}-16$ & $2.44 \mathrm{e}-15$ \\
\hline & \multicolumn{3}{|c|}{$p_{1}=0.0293, p_{2}=0.0248, n=0.2266$} \\
\hline & $\mathbf{G}$ & $\mathbf{X}$ & $T$ \\
\hline $\mathrm{TP}_{\mathrm{T} 1 \mathrm{DM}}$ & 0.728 & $6.059 \mathrm{e}-16$ & 0 \\
\hline $\mathrm{TP}_{\mathrm{T} 2 \mathrm{DM}}$ & 0.728 & $2.923 \mathrm{e}-16$ & $1.776 \mathrm{e}-15$ \\
\hline
\end{tabular}

\section{CONCLUSIONS}

The paper examined the utilization of the TP model transformation in case of T1DM and T2DM models. We demonstrated that TP models can perfectly mimic the original nonlinear systems behavior over time beside given initial values and inputs. Moreover, we investigated the robustness of the realized TP models from parameter variation point of view. Since the TP model transformation can be easily used for LMI-based controller design, this property can be useful in guaranteeing the controller's robustness by the created robust TP model. Our further work will focus investigating how can we use in practice the realized TP models from controller design point of view.

\section{ACKNOWLEDGMENT}

The authors thankfully acknowledge the support of the Robotics Special College of Obuda University. The research was supported by the European Research Council Starting Grant ERC-StG 679681.

its use to design and assess improved insulin therapies for diabetes," Ph.D. dissertation, MIT, USA, 1985.

[5] L. Kovacs, B. Benyo, J. Bokor, and Z. Benyo, "Induced 12-norm minimization of glucose-insulin system for type i diabetic patients," Comput Meth Prog Bio, vol. 102, pp. 105 - 118, 2011.

[6] P. Colmegna and R.S.S. Pena, "Analysis of three t1dm simulation models for evaluating robust closed-loop controllers," Comput Meth Prog Bio, vol. 113, pp. $371-382,2014$.

[7] P. Baranyi, D. Tikk, Y. Yam, and R. Patton, "TP model transformation as a way to lmi-based controller design," Comput Ind, vol. 51, no. 3, pp. $281-297,2003$. 

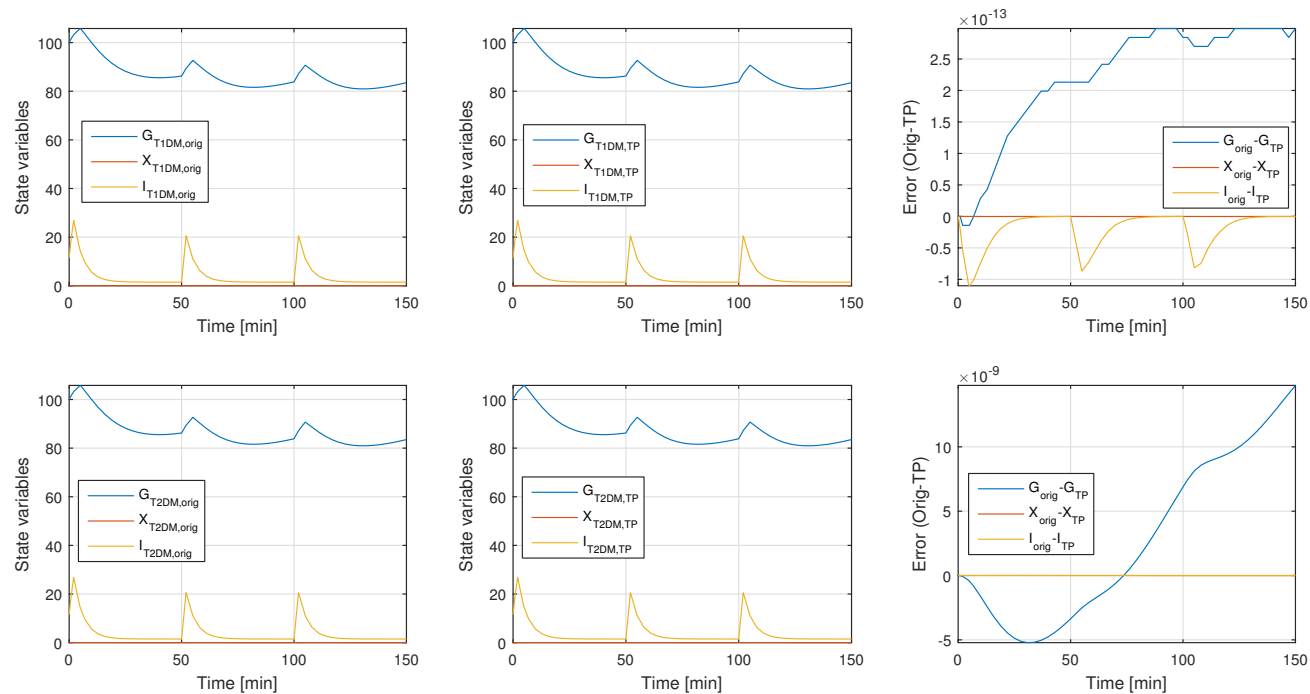

Figure 3. Comparison of the original nonlinear models and the TP versions of them; simple model case. Upper row: T1DM models; Lower row: T2DM models.
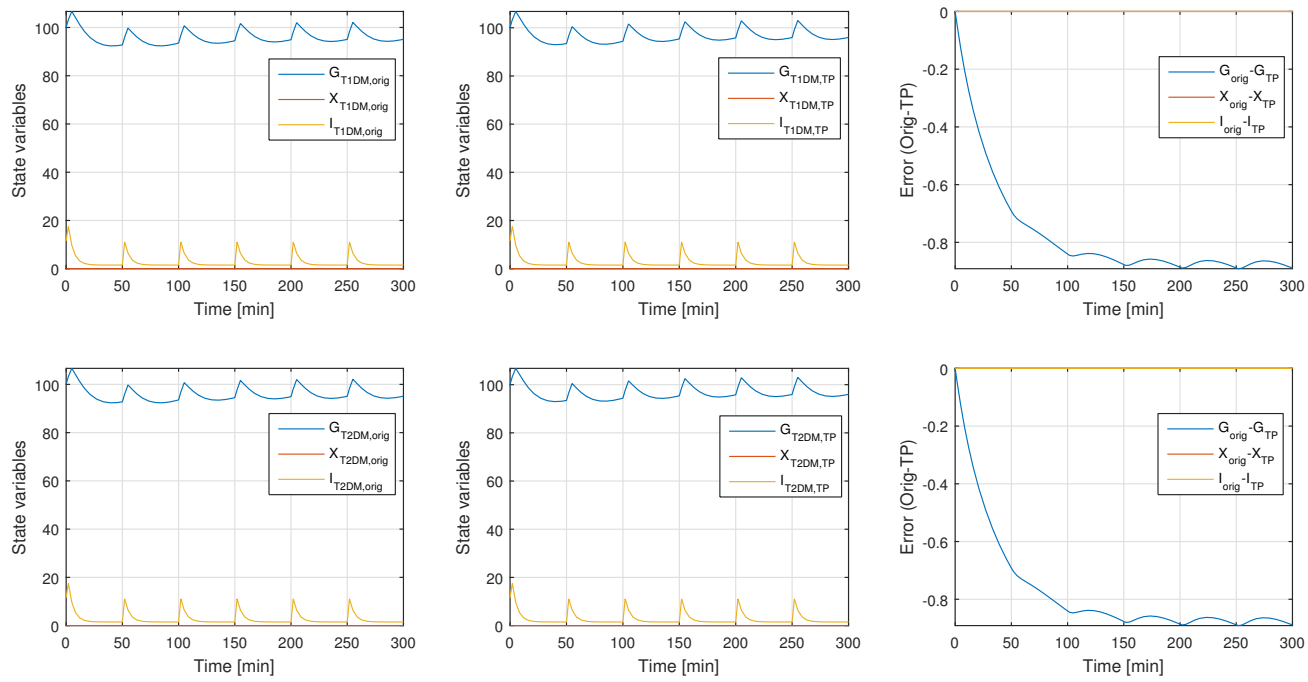

Figure 4. Comparison of the original nonlinear models and the TP versions of them; robust model case. Upper row: T1DM models; Lower row: T2DM models. Parameters: $p_{1}=0.0266, p_{2}=0.0258, n=0.2231$

[8] P. Baranyi, P. Korondi, and K. Tanaka, "Parallel distributed compensation based stabilization of a 3-DOF RC Helicopter: A tensor product transformation based approach," JACIII, vol. 13, pp. 25 - 34, 2009.

[9] P. Baranyi, Y. Yam, and P. Varlaki, Tensor Product Model Transformation in Polytopic Model-Based Control, 1st ed., ser. Series: Automation and Control Engineering. Boca Raton, USA: CRC Press, 2013.

[10] J. Kuti, P. Galambos, and P. Baranyi, "Minimal volume simplex (mvs) convex hull generation and manipulation methodology for tp model transformation," Asian J Control, 2015, submitted.

[11] F. Chee and T. Fernando, Closed-Loop Control of Blood Glucose. Heidelberg, Germany: Springer, 2007.

[12] P. Baranyi, "TP model transformation as a way to lmi-based controller design," IEEE T Ind Electron, vol. 51, no. 2, pp. 387 - 400, 2004.

[13] R. Precup, C. Dragos, S. Preitl, M. Radac, and E. Petriu, "Novel tensor product models for automatic transmission system control," IEEE Systems Journal, vol. 6, pp. 488 - 498, 2012.
[14] S. Kuntanapreeda, "Tensor product model transformation based control and synchronization of a class of fractional-order chaotic systems," Asian J Control, vol. 17, pp. 371 - 380, 2015.

[15] K. Wu and Y. Yam, "Control stability of tp model transformation design via probabilistic error bound of plant model," in Proceedings of 2013 IEEE International Conference on Systems, Man, and Cybernetics SMC 2013. IEEE SMCS, 2013, pp. 1259 - 1264.

[16] J. Kuti, P. Galambos, and P. Baranyi, "Minimal volume simplex (mvs) approach for convex hull generation in tp model transformation," in 2014 18th International Conference on Intelligent Engineering Systems (INES 2014). IEEE Hungary Section, pp. 187 - 192.

[17] P. Galambos and P. Baranyi, "TP ${ }_{\tau}$ model transformation: A systematic modelling framework to handle internal time delays in control systems," Asian J Control, vol. 17, no. 2, pp. 1 - 11, 2015.

[18] MTA SZTAKI. (2016) TPtool - Tensor Product MATLAB Toolbox. [Online]. Available: http://tptool.sztaki.hu 